\title{
How the surveillance system may bias the results of analytical epidemiological studies on BSE: prevalence among dairy versus beef suckler cattle breeds in France
}

\author{
Christian DUCROT ${ }^{\mathrm{a} *}$, Pascal ROY ${ }^{\mathrm{b}}$, Eric MORIGNAT $^{\mathrm{c}}$, Thierry BARON ${ }^{\mathrm{c}}$, \\ Didier CALAVAS ${ }^{\mathrm{C}}$
}

\author{
a Unité d'Épidémiologie Animale, INRA Theix, 63122 Saint-Genès-Champanelle, France \\ b Service de Biostatistique des Hospices Civils de Lyon, Centre Hospitalo-Universitaire Lyon-Sud, \\ 69495 Pierre-Bénite Cedex, France \\ c Unité d’Épidémiologie, AFSSA Lyon, 31 av. T. Garnier, 69364 Lyon Cedex 07, France
}

(Received 20 June 2002; accepted 10 October 2002)

\begin{abstract}
Until recently, epidemiological studies on Bovine Spongiform Encephalopathy (BSE) were based on Mandatory Reporting Systems (MRS) of clinically suspect bovines only, but rapid diagnostic tests were validated in 1999 and are used for targeted surveillance in Switzerland, France and other countries, as a complementary and secondary tool. Data on 30491 cattle issued from a French pilot program targeted at cattle having died on the farm, subjected to euthanasia or sent for emergency slaughter, did not show any significant difference in BSE risk between dairy and beef suckler breeds. The data also revealed that part of the clinical cases of BSE escaped the MRS, which permitted to detect more dairy than beef suckler affected cattle compared to the targeted surveillance in the same period (from August to December 2000) and region (Bretagne, Pays de la Loire and Basse Normandie regions). Analyzing together the data of the targeted surveillance and mandatory reporting system programs with a non-conditional logistic regression, we found that the odds of a dead cow being a BSE case among all dead cattle was 3.2 times higher for dairy breeds compared to beef suckler breeds. This confirmed British findings but points out to the fact that considering either MRS or targeted surveillance data alone may possibly create biases in epidemiological studies on BSE.
\end{abstract}

BSE / epidemiology / bias / logistic regression / breed

\section{INTRODUCTION}

Until 1999, the detection of Bovine Spongiform Encephalopathy (BSE) cases was only based on Mandatory Reporting Systems (MRS), and most epidemiological studies have been carried out on these data, including the analysis of the difference in

\footnotetext{
* Correspondence and reprints

Tel.: (33) 4736242 63; fax (33) 4736245 48; e-mail: ducrot@ clermont.inra.fr
} 
risk between dairy and beef suckler cattle. Recently, targeted surveillance (TS) programs using the Prionics test ${ }^{\circledR}$ have started on dead or slaughtered animals, as a complement to MRS that remains the first and priority detection device applied to live animals. TS programs detect clinical cases that passed through the MRS and is assumed to detect some preclinical cases as well.

With the same background as the TS program, started in 1999 in Switzerland using the Prionics test ${ }^{\circledR}[5,6]$, a TS program was carried out exhaustively in France in 2000 and during six months on cattle at risk for BSE (found dead on the farm, subjected to euthanasia or emergency slaughtered), in three contiguous regions of western France [3]. The results showed that the MRS did not detect all the clinical cases of BSE; several escaped identification, although at the clinical stage of the disease, and were only detected thanks to the TS program. Secondly, no significant difference was found in the prevalence of Prionics positive cattle between cattle from dairy herds and those from beef suckler herds in the tested population [11]. This finding was in contradiction with British data since Wilesmith [18] found that the hazard ratio of being an affected herd was three times higher for dairy herds than for beef suckler herds, and 1.7 times higher for mixed herds, in agreement with previous studies $[14,17]$.

This difference in risk between cattle from dairy and beef suckler herds is an important issue in our understanding of BSE transmission to cattle. Epidemiological studies carried out in Great Britain [16] indicate that the primary vehicle of BSE transmission to cattle is Meat and Bone Meal. Since there are different feeding practices between dairy and beef suckler herds, the use of purchased feedstuffs being on average more frequent and of greater importance for dairy cattle [8], one expects a higher risk for BSE for this cate- gory. However, this difference in risk between dairy and beef suckler herds may vary between countries, due to variations in farming practices as well as in the use of Meat and Bone Meal in the feedstuff formula [8]. Furthermore, it should depend on the period of life and feedstuff type that are the most at risk for BSE transmission, that are not yet precisely known, growth feedstuff being used in a more comparable manner between dairy and beef suckler cattle than feedstuff for adults.

In order to investigate more deeply the difference in BSE risk between dairy and beef suckler herds in the French situation, following the preliminary negative findings with the TS, we postulated a possible bias due to the detection system and analyzed all the BSE cases found with either the MRS or the TS program.

\section{MATERIALS AND METHODS}

\subsection{Definition of BSE cases}

Detected BSE cases included two types of animals; first were clinically suspect animals [13] confirmed at the national reference laboratory (AFSSA Lyon, France) using western blot [10] or immunohistochemistry [4] - i.e. cases found with the MRS - Second were Prionics positive animals confirmed with the same two techniques, among the whole cattle population found dead on the farm, subjected to euthanasia or emergency slaughtered in the geographical area and period of interest - i.e. cases found with the TS. The rationale of putting together both subpopulations is based on the fact that the estimated parameters are the proportion of BSE cases among the "population at risk for BSE" composed of dead cattle as well as cattle subjected to euthanasia. The TS was targeted at this population of interest, and the clinically suspect animals came under the same category by extrapolation because 
BSE is a fatal disease in a short delay after the clinical onset.

\subsection{Study design and reference population}

The study was carried out in the geographical area and during the period when both the MRS and the TS programs were running. It took place in three regions of western France (Bretagne, Basse Normandie and Pays de la Loire), from August 7 to December 22, 2000. The population of interest was three categories of cattle: those having died on the farm, subjected to euthanasia, and emergency slaughtered; it included both the sample of interest of the TS program [11] - i.e. 30491 cattle aged two years or more and 110 cattle reported through the MRS that displayed suspect clinical signs, and hence were subjected to euthanasia and tested for BSE. Sampling techniques and data collection are detailed in [11], and the design of the MRS in [13]

\subsection{Statistical analysis}

The explanatory variable of interest was the breed of the cattle (classified in the dairy type, beef suckler type, mixed), this data being more accurately reported on the questionnaire than the type of production of the farm (dairy operation versus beef cattle operation). In fact, both variables did not give exactly the same information, since some dairy breed cows are found on beef farms, aimed, for example, at suckling motherless calves, and some beef breed cows in dairy operations, as a complementary income. First, the proportion of detected BSE by breed, birth cohort, region of origin and death category was computed. A Chi square test was done to check whether the proportion of BSE was different between the classes of the different factors. Then a non-conditional logistic regression [1] was performed to analyze the effect of breed adjusting for the other factors. A contribution of the different factors was measured with the Odds ratio. The factors included in the analysis were the breed (dairy, beef suckler, mixed or not reported), the region of the farm (BasseNormandie, Bretagne, Pays de la Loire), and the birth cohort $(<93,93-94,94-95$, 95-96, 96-97, >97). Birth cohorts were grouped by seasonal year of birth (from July 1st to June 30th) except for the first and the last group that pooled several seasonal years with a small sample size. Second-degree product terms were then introduced to test the interaction. The data management was done using ACCESS (Microsoft Access 97 SR-2, Copyright (C) 1989-1997 Microsoft Corporation) and the statistical analysis using S-Plus software (S-Plus 2000 Professional Release 2, Copyright (C) 1998-1999 Mathsoft, Inc., New-York, USA).

\section{RESULTS}

\subsection{Characteristics of the BSE cases depending on the detection system}

In the area and period of interest, we first compared the production type of the farms where BSE cases were found using the MRS to those found using the TS program. The MRS identified 34 BSE cases, all from dairy herds, while the TS program discovered 49 cases, with only $36(73.5 \%)$ from dairy herds (Fig. 1). This partial finding has to be verified on a larger scale but it suggests that the ability of the MRS used on live animals and before the TS screening - to detect BSE clinical suspects might depend on the breed of the animals, dairy versus beef suckler. In our study, it could have biased the observed effect of the breed of the animals on the risk of BSE, given that the BSE cases were not distributed independently from the variable of interest - the breed of the animals - in the two detection programs. 


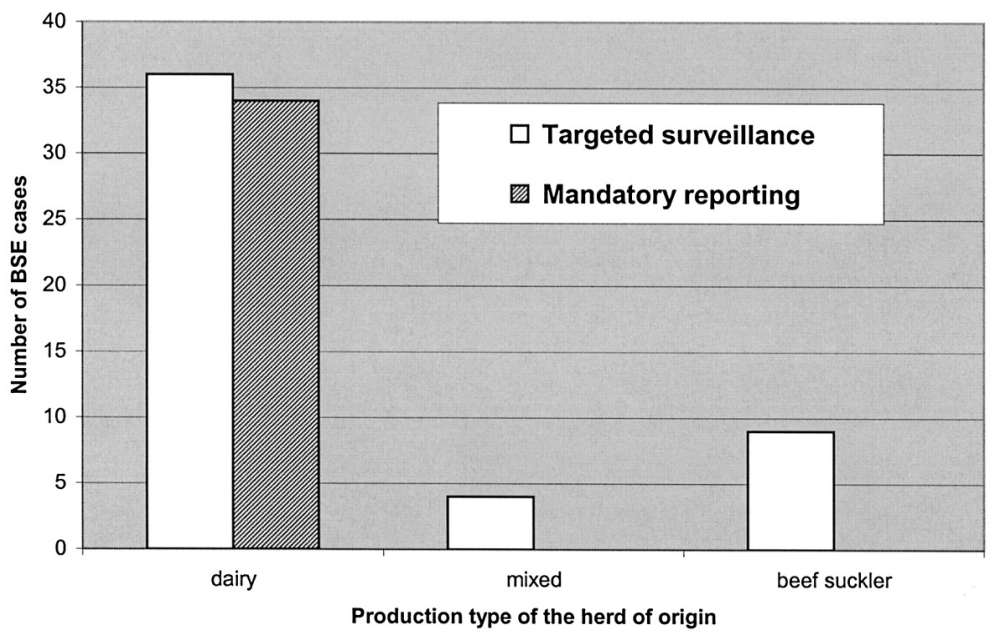

Figure 1. Distribution of the BSE cases depending on the detection program.

From August 7 to December 22, 2000, in Western France, an overall number of 83 BSE cases were detected. Seventy were from a dairy herd, nine from a beef suckler herd, and the last four from a mixed or unknown type of herd. The type of herd (dairy versus beef) is partly different from the breed type of the positive animal that is presented in Table I (dairy versus beef breed), dairy herds breeding sometimes a few beef cows and conversely.

\subsection{Difference in BSE risk between dairy and beef suckler cattle}

For this reason, using either the data from the MRS alone or the data from the TS program alone was inappropriate to measure the difference in BSE risk between dairy and beef suckler cattle. We thus simultaneously took into account the data from the two detection programs running altogether - to perform the analysis on the area and period of interest. Among the 30601 cattle found dead on the farm, subjected to euthanasia or emergency slaughtered (including 110 clinically suspect animals), the overall detection rate of BSE was 2.7 per thousand. It was respectively 3.3 per thousand among dairy breed cattle, 0.9 per thousand among beef suckler breed cattle and 1.3 per thousand among animals from mixed or unknown breeds; the difference was statistically significant (Chi $2=11.1 ; 2$ degrees of freedom; $p=0.0038$ ).
The detection rate of BSE was also significantly different according to the year of birth (Chi $2=175.8 ; 5$ degrees of freedom; $p<0.0001)$ and the geographical area (Chi $2=14.4 ; 2$ degrees of freedom; $p=$ 0.0007). Adjusting for these two variables, the association between the breed and the detection rate of BSE remained significant (Tab. I). Furthermore, none of the interaction terms was significant, and they were removed from the model. The odds of BSE among dead cattle was 3.2 times higher (95\% confidence interval: $1.16 ; 8.9)$ for the dairy breeds compared to the beef suckler breeds.

\section{DISCUSSION}

As expressed by Heim [9], MRS alone is not sufficient to derive a true picture of the BSE status in a country, because such reporting is too dependant on subjective factors. More precisely, BSE is difficult to 
Table I. Description of the cattle involved in the Mandatory Reporting System and Targeted Surveillance Program in Western France from August 7 to December 22, 2000, adjusted odds ratio (OR) and $95 \%$ confidence interval (CI) from a multivariable non conditional logistic regression on the risk factors for the proportion of detected BSE among cattle at risk.

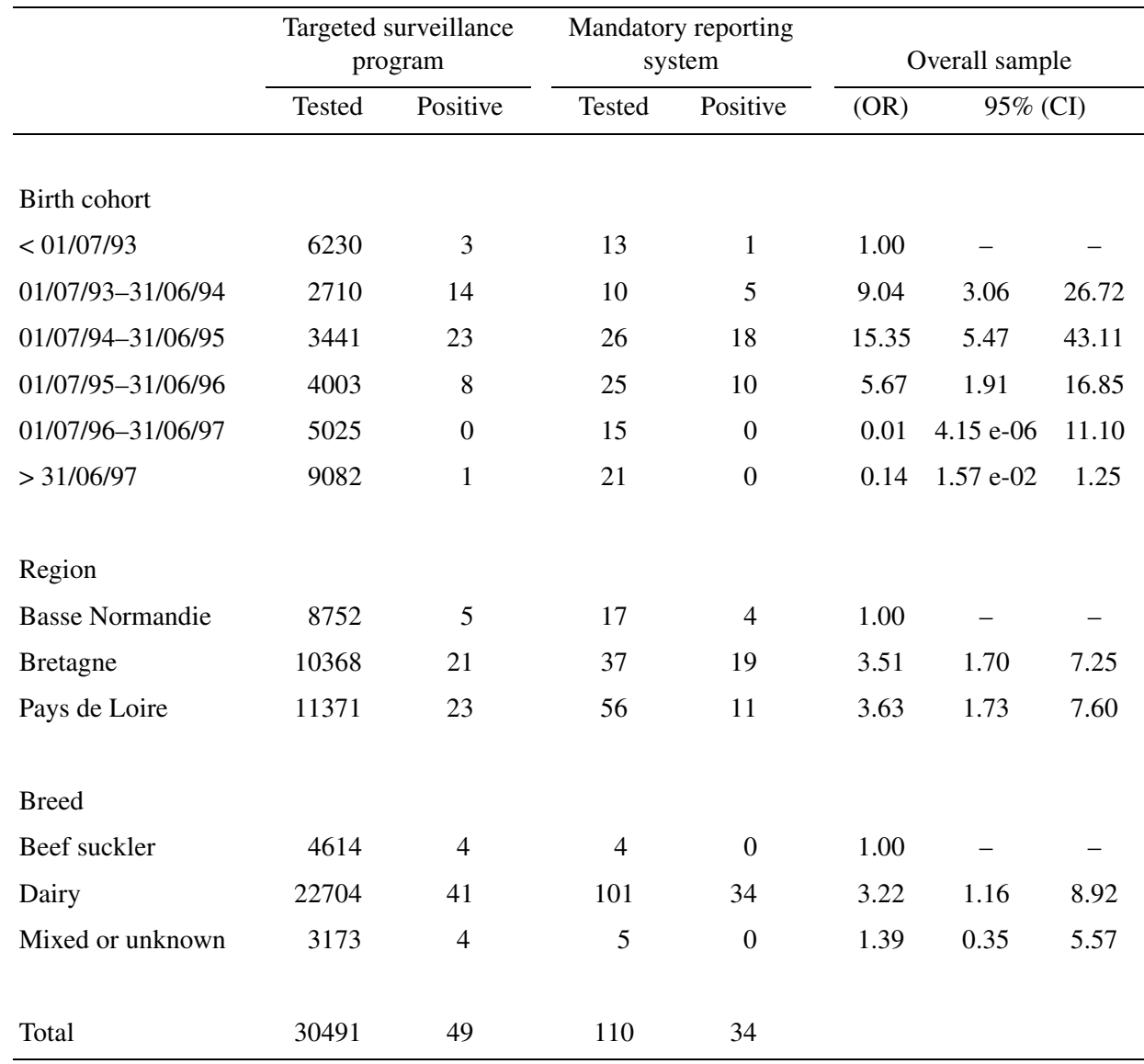

The residual deviance of the model is 984.7 , with 30591 degrees of freedom; the difference of deviance compared to the constant model is 162.2 ( 9 degrees of freedom). The improvement provided by each variable in this model has been measured by the difference of variance between the final model and the same without the studied variable. The difference of deviance (degrees of freedom) is 133.1 (5) for the birth cohort, 16.0 ( 2 degrees of freedom) for the region, and 8.3 (2) for the breed.

diagnose clinically for various reasons. Affected cows do not always present the typical signs of the disease and clinical signs taken individually do not have a good positive predictive value for the diagnosis (unpublished results). Also, it may occur, for mature animals reaching the end of their productive life, that culling without resorting to a diagnosis may be considered the most expedient clinical decision. Finally, this is a rare and relatively novel disease so we lack experience and awareness among cattle owners, herdsmen and veterinary surgeons. The difference between beef and dairy cattle in the efficiency of the MRS to detect BSE has to be confirmed at a larger scale. It may partly be due to a lower awareness and vigilance 
concerning beef cattle, that has been evidenced with a vigilance index (unpublished results). Furthermore, clinical signs could be less marked in beef suckler cows than in dairy cows because they are subject to generally less observation and less stimuli, notably those resulting from the process of milking, which can make the clinical signs more pronounced or noticeable.

In this context, TS systems based on rapid tests [12] have proven to be a complementary tool for BSE detection $[3,6]$. Pooling together MRS and TS cases seems reasonable since we defined the cases as positive testing animals among the dead population; it includes clinical cases as well as preclinical cases that reached the end of the incubation period - on the basis of pathogenesis trials [15]. Cases found with MRS were clinically affected animals [13]. If they had not been detected through MRS, they would have died within a short delay, since BSE is a fatal disease, and would have been detected through the TS program. A retrospective clinical investigation [2] showed that this has been the case for part of the positive animals found in the TS program. This investigation was carried out on every positive case by a separate interview of the farmer and of the veterinarian practitioner. It appeared that $68 \%$ of the BSE cases found with the screening test had already displayed clinical signs before death, either characteristic of BSE or sufficiently suggestive, which is consistent with the Swiss data [6]. Another discussion point is the specificity of BSE detection, that is comparable for the MRS and TS programs since the confirmation tests are the same, Western blot [10] and/or immunohistochemistry [4] carried out at the reference laboratory. Concerning sensitivity, combining the MRS and TS of cattle at risk allowed to check every bovine aged two years and more at the time of death or euthanasia, which appears to be an efficient method to detect a $100 \%$ lethal disease. The only concern is that some clinically affected bovine could have been sent to the abattoir and slaughtered without any test. However, when the TS program was set up in France, this bias was pointed out and a strong and comprehensive antemortem surveillance has been organized at the abattoirs all over France, thus ensuring a reinforced surveillance of the clinical signs.

The results show a significant difference in BSE risk in the dead population depending on the cattle breed, dairy versus beef suckler, with an odds ratio equal to 3.2 (95\% CI 1.2 - 8.9). They confirmed the bias due to the detection program since there was no difference between breeds when the analysis was limited to the cases detected with the TS; with the same method, the odds ratio was then 1.25 (95\% CI 0.5 - 2.9) for dairy compared to beef cattle [11]. Our results obtained at the animal level among dead cattle cannot be compared directly to the results obtained by Wilesmith [18] at the herd level, but they show the same trend. They reinforce the general idea that feedstuffs at risk were fed in a larger amount to dairy cattle than beef suckler cattle, which can orient the inquiry about feedstuffs involved in BSE transmission.

The study was performed on dead animals because this population is at risk and the rapid tests are used post mortem. However, the real population of interest in comparing the contamination level between dairy and beef suckler cattle is the overall cattle population. We cannot extrapolate our results from the dead population to the live population because from our data we cannot infer the prevalence of positive testing animals on alive cattle. We know that the average annual rate of death among cattle is higher for dairy than for beef suckler cows; during the 4.5 month study period, the ratio of dead cattle aged two years and more per hundred adult cows (census of year 2000) - with adults being defined as animals that have calved at least once - was respectively 1.2 for dairy and 0.6 for beef cattle in the three regions of 
interest. Furthermore, the preliminary results of the European screening program show that the percentage of positive animals is much higher on dead cattle or cattle subjected to euthanasia than on cattle slaughtered for consumption, i.e. healthy cattle. Also, a preliminary analysis of the French abattoir data shows that dairy cattle present a far higher BSE prevalence than beef cattle at culling [7]. From these data, we can postulate that the real difference in BSE contamination between dairy and beef suckler cattle should be higher than the difference in prevalence estimated from the population at risk. The detailed analysis of the data issued from the BSE screening program carried out at the abattoir since January 2001 will produce more knowledge on the proportion of apparently healthy cattle that prove to be test positive.

The results of this study confirmed the epidemiological bias due to the fact that MRS was more efficient in detecting dairy cases of BSE than beef suckler cases in 2000; thus it hid the association between breed and BSE risk within the TS program. Such findings point out the possibility of other biases in epidemiological studies due to the fact that the efficiency of MRS might differ depending on certain parameters, like region or year for example. The complementary use of MRS and TS programs should improve the reliability of epidemiological studies on BSE, but even more precise and unbiased data will be derived from the development of ante-mortem tests.

\section{ACKNOWLEDGEMENTS}

The authors thank the French Ministry of Agriculture and Fisheries that funded and has been in charge of the overall logistic aspects of these studies, and the Scientific steering committee of the TS program: Dr. Girard, CERVI, Lyon, France; Pr. J. Brugère-Picoux, ENVA, Maisons-Alfort, France; Dr. D. Costagliola, INSERM SC4, Paris, France; Dr. J.-C. Desenclos, InVS, Saint-Maurice, France; Pr. M. Eloit, ENVA, Maisons-Alfort, France; Dr. D. Heim, Dr. L. Perler, Office vétérinaire fédéral, Liebefeld, Switzerland; Dr. F. Moutou, AFSSA, Maisons-Alfort, France; Dr. A. Tibi, Pharmacie centrale des hôpitaux, Paris, France.

\section{REFERENCES}

[1] Breslow N.E., Statistical methods in cancer research: The analysis of case-control studies, Vol. 1, World health organization, Lyon, 1980.

[2] Calavas D., Ducrot C., Programme pilote de surveillance de l'ESB. Enquête clinique rétrospective concernant les bovins positifs, in: Afssa documents [online] (22 May 2001) http://www.afssa.fr/ftp/basedoc/ annexe2esb30mai.pdf (AFSSA, MaisonsAlfort, 2001).

[3] Calavas D., Ducrot C., Baron T., Morignat E., Vinard J.-L., Biacabe A.-G., Madec J.-Y., Bencsik A., Debeer S., Eliazsewicz M., Prevalence of BSE in western France by screening cattle at risk: preliminary results of a pilot study, Vet. Rec. 149 (2001) 55-56.

[4] Debeer S., Baron T., Bencsik A., Immunohistochemistry of PrPsc within bovine spongiform encephalopathy samples with graded autolysis, J. Histochem. Cytochem. 49 (2001) 1519-1524.

[5] Doherr M.G., Oesch B., Moser M., Vandevelde M., Heim D., Targeted surveillance for bovine spongiform encephalopathy, Vet. Rec. 145 (1999) 672672.

[6] Doherr M.G., Heim D., Fatzer R., Cohen C.H., Vandevelde M., Zurbriggen A., Targeted surveillance of high-risk cattle populations for BSE to augment mandatory reporting of clinical suspects, Prev. Vet. Med. 51 (2001) 3-16.

[7] Ducrot C., Maillot M., Morignat E., Gasqui P., Buquet B., Calavas D., Que nous ont appris les tests réalisés en abattoir depuis janvier 2001 sur la situation épidémiologique de l'ESB en France ? in: Proceedings $9^{\text {es }}$ journées des sciences du muscle et technologies de la viande, Clermont-Ferrand, France, Le Pôle Viande, 2002, pp. 193-194.

[8] Enjalbert F., Les farines de viande : intérêts dans l'alimentation des ruminants et réglementation, Le Point Vétérinaire 28 (1996) 689-695.

[9] Heim D., Wilesmith J.W., Surveillance of BSE, Arch. Virol. (2000) 127-133.

[10] Madec J.-Y., Belli P., Calavas D., Baron T., Efficiency of Western blotting for the 
specific immunodetection of proteinase K-resistant prion protein in BSE diagnosis in France, Vet. Rec. 146 (2000) 74-76.

[11] Morignat E., Ducrot C., Roy P., Baron T., Vinard J.-L., Biacabe A.-G., Madec J.-Y., Bencsik A., Debeer S., Eliazsewicz M., Calavas D., Targeted surveillance to assess the prevalence of BSE in high-risk populations in western France and the associated risk factors, Vet. Rec. 151 (2001) 73-77.

[12] Moynagh J., Schimmel H., Tests for BSE evaluated, Nature 400 (1999) 105.

[13] Savey M., Belli P., Coudert M., Le réseau d'épidémiosurveillance de la BSE en France : principes - premiers résultats, Epidémiol. Santé Anim. 19 (1991) 49-61.

[14] Stevenson M.A., Wilesmith J.W., Ryan J.B.M., Morris R.S., Lockhart J.W., Lin D., Jackson R., Temporal aspects of the epidemic of bovine spongiform encephalopathy in Great Britain: individual animal-associated risk factors for the disease, Vet. Rec. 147 (2000) 349-354.
[15] Wells G.A.H., Hawkins S.A.C., Green R.B., Austin A.R., Dexter I., Spencer Y.I., Chaplin M.J., Stack M.J., Dawson M., Preliminary observations on the pathogenesis of experimental bovine spongiform encephalopathy (BSE): an update, Vet. Rec. 142 (1998) 103106.

[16] Wilesmith J.W., Ryan J.B.M., Hueston W.D., Bovine spongiform encephalopathy: casecontrol studies of calf feeding practices and meat and bonemeal inclusion in proprietary concentrates, Res. Vet. Sci. 52 (1992) 325331.

[17] Wilesmith J.W., Ryan J.B.M., Hueston W.D., Hoinville L.J., Bovine spongiform encephalopathy: epidemiological features 1985 to 1990, Vet. Rec. 130 (1992) 90-94.

[18] Wilesmith J.W., Ryan J.B.M., Stevenson M.A., Morris R.S., Pfeiffer D.U., Lin D., Jackson R., Sanson R.L., Temporal aspects of the epidemic of bovine spongiform encephalopathy in Great Britain: holdingassociated risk factors for the disease, Vet. Rec. 147 (2000) 319-325. 